\title{
Comparative transcriptional analysis of hop responses to infection with Verticillium nonalfalfae
}

\author{
Vasja Progar $^{1} \cdot$ Jernej Jakše $^{1} \cdot$ Nataša Štajner ${ }^{1} \cdot$ Sebastjan Radišek ${ }^{2} \cdot$ \\ Branka Javornik $^{1} \cdot$ Sabina Berne $^{1}$ (D)
}

Received: 14 April 2017/ Accepted: 4 July 2017 / Published online: 11 July 2017

(c) The Author(s) 2017. This article is an open access publication

\begin{abstract}
Key Message Dynamic transcriptome profiling revealed excessive, yet ineffective, immune response to $V$. nonalfalfae infection in susceptible hop, global gene downregulation in shoots of resistant hop and only a few infection-associated genes in roots.

Abstract Hop (Humulus lupulus L.) production is hampered by Verticillium wilt, a disease predominantly caused by the soil-borne fungus Verticillium nonalfalfae. Only a few hop cultivars exhibit resistance towards it and mechanisms of this resistance have not been discovered. In this study, we compared global transcriptional responses in roots and
\end{abstract}

Communicated by Leandro Peña.

Electronic supplementary material The online version of this article (doi:10.1007/s00299-017-2177-1) contains supplementary material, which is available to authorized users.

Sabina Berne

sabina.berne@bf.uni-lj.si

Vasja Progar

vasja.progar@bf.uni-lj.si

Jernej Jakše

jernej.jakse@bf.uni-lj.si

Nataša Štajner

natasa.stajner@bf.uni-lj.si

Sebastjan Radišek

sebastjan.radisek@ihps.si

Branka Javornik

branka.javornik@bf.uni-lj.si

1 Department of Agronomy, Biotechnical Faculty, University of Ljubljana, Ljubljana, Slovenia

2 Plant Protection Department, Slovenian Institute of Hop Research and Brewing, Žalec, Slovenia shoots of resistant and susceptible hop plants infected by a lethal strain of $V$. nonalfalfae. Time-series differential gene expression profiles between infected and mock inoculated plants were determined and subjected to network-based analysis of functional enrichment. In the resistant hop cultivar, a remarkably low number of genes were differentially expressed in roots in response to $V$. nonalfalfae infection, while the majority of differentially expressed genes were down-regulated in shoots. The most significantly affected genes were related to cutin biosynthesis, cell wall biogenesis, lateral root development and terpenoid biosynthesis. On the other hand, susceptible hop exhibited a strong defence response in shoots and roots, including increased expression of genes associated with plant responses, such as innate immunity, wounding, jasmonic acid pathway and chitinase activity. Strong induction of defence-associated genes in susceptible hop and a low number of infection-responsive genes in the roots of resistant hop are consistent with previous findings, confirming the pattern of excessive response of the susceptible cultivar, which ultimately fails to protect the plant from $V$. nonalfalfae. This research offers a multifaceted overview of transcriptional responses of susceptible and resistant hop cultivars to $V$. nonalfalfae infection and represents a valuable resource in the study of this plantpathogen interaction.

Keywords Hops · Verticillium wilt · Biotic stress · RNASeq $\cdot$ Differential gene expression

\section{Introduction}

Hop (Humulus lupulus L.) is a perennial, dioecious plant belonging to the Cannabaceae family. It is cultivated commercially for hop products comprising hop cones, 
powdered or pelleted hops and hop extracts (Haunold 2010). According to the International Hop Growers' Convention (Paris, France-April 18, 2016), it was grown on 53,876 hectares in 2016 , with an estimated worldwide hop production of 105,642 metric tons. Traditionally, hop has been used in the brewing industry as an ingredient that adds characteristic bitterness and aroma to beer, as well as stabilizing the foam and preserving the beer (Steenackers et al. 2015). In recent years, hop-derived compounds have been discovered that exhibit health beneficial biological properties, including anti-oxidative, anti-inflammatory, anti-proliferative and anti-carcinogenic activities, as well as the suppression of osteoporosis and obesity (Van Cleemput et al. 2009; Kirkwood et al. 2013; Li et al. 2015).

Worldwide, hop plantations suffer economic losses due to fungal diseases, such as downy mildew, powdery mildew and Verticillium wilt. The latter disease on hop is predominantly caused by Verticillium nonalfalfae (formerly Verticillium albo-atrum), a soil-borne fungus. The disease symptoms vary depending on the strain's aggressiveness, from minor wilting symptoms (yellowing and upward curling of leaves, swollen bines and brown discolouration of vascular tissue) caused by the mild pathotype to rapid collapse of leaves and branches, resulting in plant death within a few months after infection with the lethal pathotype (Radišek et al. 2003, 2006). V. nonalfalfae released from decaying plants forms melanized hyphae as resting structures, which survive in soil for three to 4 years. On sensing host plant root exudates, these structures germinate into mycelial hyphae, which directly penetrate the root epidermal cells, enter the xylem vessels and spread systemically by the production of spores that are carried through the plant by the transpiration flow (Yadeta and Thomma 2013; Cregeen et al. 2015).

At the early stages of infection by vascular plant pathogens, plant defence relies on protective physical barriers (e.g., Casparian strips, suberin), preformed chemical defence compounds (e.g., glucosinolates, flavonoids, antimicrobial proteins) and general immune responses induced by microbe-associated molecular patterns (MAMPs) (De Coninck et al. 2014). Once the pathogens reach the xylem vessels, they are presumably recognized by specific extracellular receptors in the surrounding parenchyma cells. This recognition activates the formation of tyloses, the accumulation of pectin-rich gels and gums, vascular coating and callose and secondary cell wall depositions (Yadeta and Thomma 2013; De Coninck et al. 2014; Cregeen et al. 2015). A tissue-specific developmental program that leads to the formation of new xylem elements is also observed (Reusche et al. 2012). Furthermore, significant metabolic changes occur and involve the induction of pathogenesis-related (PR) proteins, peroxidases and proteases, as well as the production of phytoalexins, sulphur-containing compounds and phenolic compounds (Yadeta and Thomma 2013; De Coninck et al. 2014). Plant microarray and RNA-Seq studies have revealed that the interaction between vascular wilt pathogens and host plants involves transcriptional reprogramming of hundreds of genes, activation of $\mathrm{Ca}^{2+}$-signalling pathways, induction of ROS and MAPK cascades and modulation of phytohormone signalling ( $\mathrm{Hu}$ et al. 2008; van Esse et al. 2009; Chen et al. 2014).

Transcriptomic studies on compatible and incompatible interactions between Verticillium spp. and several hosts have been reported employing data generated by RNAseq. Comparison of the sea-island cotton (resistant) and upland cotton (susceptible) response to infection with aggressive and mild strains of $V$. dahliae led to the identification of 44 differentially expressed genes (DEGs) with possible implication in the resistance reaction, highlighting genes involved in the phenylpropanoid pathway (Sun et al. 2013), similar to other findings in cotton (Xu et al. 2011), tomato (Gayoso et al. 2010) and wild eggplants (Zhou et al. 2016), in which synthesis of lignin is presented as an important plant defence reaction against infection by Verticillium species. Most DEGs found in the compatible interaction of the tomato- $V$. dahliae pathosystem were also associated with phenylpropanoid metabolism (Tan et al. 2015). In the compatible interaction of Medicago truncatula and Verticillium alfalfae, Toueni et al. (2016) found a disorganized response involving many genes from different functional classes. In contrast, in the incompatible interaction, they identified several genes associated with PTI and hormonal signalling, as well as several transcriptional factors, suggesting that the resistance of the studied M. truncatula line is due to innate immunity. Global transcriptome profiling and DEGs analysis were also carried out on sunflower- $V$. dahliae interactions, revealing massive transcriptional reprogramming and classifying resistant cultivar DEGs into plant hormone signalling transduction, plant-pathogen interaction and flavonoid biosynthesis functional categories (Guo et al. 2017).

Although several fungicides have been found to be effective against Verticillium spp. in vitro, none have proved successful in field tests (Crowe and Parks 2001). To date, the only measures to control Verticillium wilt are phytosanitary measures, crop rotation and planting resistant crop varieties. In tomato, a leucine-rich repeat receptor-like protein $\mathrm{Ve} 1$ was shown to confer resistance against race 1 strains of $V$. dahliae (Fradin et al. 2009), which secrete the Ave1 effector protein (de Jonge et al. 2012). In the following years, several Ve1 homologs were identified within and outside the Solanaceae family, indicating an ancient origin of this immune receptor (Song et al. 2016). A functional hop homolog of the immune receptor Ve1 has been reported to mediate resistance against $V$. dahliae but 
triggers only a weak hypersensitive response when co-expressed with Ave1 in Nicotiana tabacum (Song et al. 2016). However, the Ave1 sequence could not be found in hop-infecting strains of $V$. nonalfalfae (unpublished data) and so the $\mathrm{Ve} 1$ receptor does not seem to convey resistance to this pathogen in hop.

Understanding the mechanisms of hop resistance to Verticillium wilt and the identification of the participating genes would facilitate marker-assisted selection in hop resistance breeding. Genetic studies of Verticillium wilt resistance in hop have revealed a single genomic QTL region associated with resistance to $V$. nonalfalfae, explaining up to $26 \%$ of phenotypic resistance and suggesting the involvement of more than a single gene (Jakše et al. 2013). In a proteomic study of the hop-V. nonalfalfae pathosystem, an accumulation of defence-related proteins was detected in the roots of a susceptible cultivar but no significant infection-specific changes were observed in the roots of a resistant cultivar, indicating the involvement of constitutive rather than induced defence mechanisms in resistance to $V$. nonalfalfae (Mandelc et al. 2013). A transcriptional study on the same pathosystem confirmed strong up-regulation of genes for pathogenesis-related proteins in the susceptible cultivar, while genes implicated in ubiquitination and vesicle trafficking were reported to be up-regulated in the resistant and down-regulated in the susceptible cultivar (Cregeen et al. 2015).

In this study, we investigated the dynamics of hop- $V$. nonalfalfae interactions to identify specific defence responses of susceptible and resistant hop cultivars. To demonstrate this, we set up an experiment in which susceptible and resistant hop plants were inoculated with a lethal strain of $V$. nonalfalfae and compared to mockinoculated samples of shoots and roots at four time points. The samples were subjected to RNA-Seq profiling, differential expression analysis and functional network analysis. The results indicate that the susceptible hop cultivar mounts a strong basal defence response against $V$. nonalfalfae, while in the resistant cultivar there was a remarkably low differential expression in roots and a strong general down-regulation of genes in shoots. In addition to providing a global overview of the response of susceptible and resistant hop plants to $V$. nonalfalfae, the data generated in the course of the study will serve as an invaluable resource for research of Verticillium wilt.

\section{Materials and methods}

\section{Plant material and colonization}

The study was performed on a Verticillium wilt resistant hop cultivar (Wye Target) and a susceptible cultivar
(Celeia). One-year-old rooted cuttings of both hop cultivars were inoculated with a lethal PV1 pathotype (T2 isolate) of $V$. nonalfalfae by ten-minute root-dipping in a fungal spore suspension $\left(5 \times 10^{6}\right.$ conidia per $\left.\mathrm{ml}\right)$, while control plants were mock inoculated using sterile water (Flajšman et al. 2017). After root-dipping, the plants were transplanted to a sterile commercial substrate and grown under controlled conditions in a plant growth chamber with a $12 \mathrm{~h}$ photoperiod at $22{ }^{\circ} \mathrm{C}$ and $65 \%$ relative humidity during the light period, and $20{ }^{\circ} \mathrm{C}$ and $70 \%$ relative humidity during the dark period.

The experimental setup is illustrated in Fig. 1. Root and shoot samples of susceptible and resistant plants were harvested at 6, 12, 18 and 30 days post inoculation. For each condition, both inoculated and mock inoculated plants were collected to be compared for differential expression. Samples from three individual hop plants per condition were ground in liquid nitrogen and stored at $-80{ }^{\circ} \mathrm{C}$. To confirm $V$. nonalfalfae infection in the sampled plants, mycological re-isolation of the pathogen was carried out and the presence of fungal DNA was additionally confirmed with qPCR, as described previously (Cregeen et al. 2015).

\section{RNA isolation}

Total RNA was isolated with a Spectrum Plant Total RNA Kit (Sigma-Aldrich) in combination with on-column DNase treatment (On-Column DNase I Digestion Set, Sigma-Aldrich). The concentration and purity were measured on a NanoVue spectrophotometer (GE Healthcare). The quality of isolated RNA was further assessed on the Agilent 2100 Bioanalyzer system (Agilent Technologies) using an Agilent RNA 6000 Nano Kit (Agilent Technologies).

susceptible hop (Celeia) / resistant hop (Wye Target)

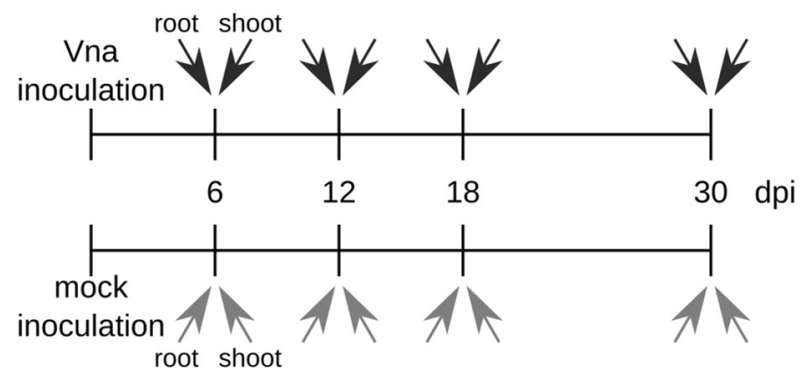

Fig. 1 Study design-sampling scheme. The samples were collected from susceptible (Celeia) and resistant (Wye Target) hop cultivars, at 4 time points, for roots and shoots separately. Plants at each condition were either inoculated with $V$. nonalfalfae or mock inoculated, thus obtaining 32 different samples in total 


\section{Sequencing}

For sequencing, total RNA from 3 biological replicates per condition was pooled in equal amounts $(1 \mu \mathrm{g}$ per replicate), thus obtaining 32 different samples (4 time points $* 2$ cultivars $* 2$ tissues $* 2$ control/inoculated). mRNA libraries were constructed using an Illumina TruSeq Sample Prep Kit and sequenced on the Illumina Genome Analyzer HiSeq 2000 platform, using a 50 bp single-end read setup at a minimum depth of 20 million reads per sample. The resulting raw nucleotide sequences have been deposited for public availability in the European Nucleotide Archive, collectively under study ID PRJEB14243 (http://www.ebi.ac.uk/ena/data/view/PRJEB14243).

\section{RNA-Seq mapping and functional annotation}

The RNA-Seq pipeline was implemented in CLC Genomics Server 7.0.2 (Qiagen; https://www.qiagenbioinfor matics.com/products/clc-genomics-server/) and consisted of trimming low-quality, adaptor-contaminated and underlength sequenced reads and mapping of processed reads (corresponding to a minimum of $90 \%$ read length with at least $90 \%$ similarity) to the nucleotide sequences of reference draft hop genome natsume.shinsuwase.v1.0.11262014 (HopBase: A unified genomic database for hop; http:// hopbase.cgrb.oregonstate.edu/). Exact trimming and mapping parameters are given in Online Resource 1. The sequence data were also examined against the Verticillium genome to ensure that the data were not skewed to expressed genes that share conservation between hop and Verticillium.

Functional annotation of gene models from the reference genome was performed with a blastx search (Altschul et al. 1997) against the Viridiplantae section of the NCBI $n r$ database and by subsequent Blast2GO analysis (Conesa et al. 2005) to assign them Gene Ontology (GO) terms, which were then further mapped to GO Slim Plant terms. Additionally, a blastx search against the TAIR10 representative database (TAIR: The Arabidopsis Information Resource; https://www.arabidopsis.org/) was performed to associate gene models with TAIR IDs of best matching Arabidopsis hits. Gene models were mapped to KEGG Arabidopsis pathways based on their associated TAIR IDs.

\section{Library normalization and determination of differential gene expression}

Each of the 32 samples was represented as a library of read counts mapped to 46,735 hop gene models. Read counts were processed using customized scripts in the R software environment (The R Project for Statistical Computing, https://www.r-project.org/). The libraries were first adjusted for composition bias by the trimmed mean of the $M$ values normalization (TMM) method (Robinson and Oshlack 2010) using the edgeR package (Robinson et al. 2010) and for size by computing CPM (counts per million-number of reads mapped to a gene model per million reads mapped to the library). Only gene models with CPM $>1$ in at least one of the samples were considered expressed (23,755 gene models), the remainder were filtered out. To facilitate further analysis, the data were represented as a matrix of $\log _{2} \mathrm{CPM}$ expression values.

Differential gene expression was estimated by computing the area of the region bounded by time series expression profiles of infected and control (mock inoculated) plants, from which the significance of differential expression was determined according to the method described by Di Camillo et al. (2007). The method was applied to all four cultivar-tissue combinations (resistant shoot, resistant root, susceptible shoot and susceptible root). The calculations, ranking of genes and statistics for this method were performed using the $\mathrm{R}$ package FunPat (Sanavia et al. 2015). An expression level dependent model was used to estimate the error, and the quantiles of the empirical distribution were chosen for a null hypothesis distribution. The obtained $p$ values were adjusted for multiple testing using the default FunPat method. In each cultivar-tissue combination, the genes were considered as differentially expressed genes (DEGs) if $p_{\text {adj. }}<0.05$. The results of the FunPat analysis are included in Online Resource 2.

Enrichment of individual cultivar-tissue combinations with GO Slim Plant terms and KEGG pathways was assessed by considering the number of DEGs that were assigned to an individual GO term or KEGG pathway relative to the total number of DEGs in that cultivar-tissue combination.

\section{Network-based analysis of functional enrichment}

We estimated the functional group enrichment of the most differentially expressed genes using a Cytoscape plugin ClueGO (Bindea et al. 2009). For each cultivartissue combination, the top 100 DEGs were selected according to their rank assigned by FunPat analysis; for roots of the resistant cultivar, some genes with $p_{\text {adj. }}>0.05$ were included to allow comparison with the other combinations. TAIR IDs associated with these genes were used as an input to ClueGO, in which Gene Ontology (GO) terms- and group-enrichment analysis was performed at medium network specificity (GO levels between 3 and 8 ) using the one-sided hypergeometric test with Bonferroni step-down $p$ value correction for multiple testing. 


\section{Identification of significant temporal profiles of differentially expressed genes}

To identify significant temporal differential expression profiles of the top 100 DEGs in a time course of each cultivar-tissue combination (the same subset of DEGs as used for network-based enrichment analysis), their $\log _{2}$ fold-change $\left(\log _{2} \mathrm{FC}\right)$ values for each time point $(6,12,18$ and 30 days post inoculation) were subjected to STEMShort Time-series Expression Miner software (Ernst and Bar-Joseph 2006). The program was set to assign DEGs to the best fitting temporal profiles, with the following parameters: the maximum number of model profiles was set to 50 , the maximum unit change in model profiles between time points to 5 and maximum correlation of model profiles to 0.8 . For statistical evaluation, all permutations were used for each DEG and $p$ values were adjusted for multiple testing with the Bonferroni correction. Cross references of the DEGs with their associated TAIR IDs were provided to the program to determine the enrichment of significant temporal profiles with GO terms, using TAIR as the gene annotation source. The analysis was performed on GO biological process terms with levels between 3 and 5 and a randomization test with 1000 samples was applied to adjust $p$ values for multiple testing.

\section{Validation of gene differential expression patterns with reverse transcription quantitative PCR}

The expression patterns determined by RNA-Seq were validated with reverse transcription quantitative PCR (RTqPCR) on twelve selected genes. Primers for these genes were designed with Primer3 software (Untergasser et al. 2012) and are listed in Online Resource 1. cDNA synthesis was performed from $1 \mu \mathrm{g}$ of total RNA in $20 \mu \mathrm{l}$ reactions using a High-Capacity cDNA Reverse Transcription Kit (Applied Biosystems). The qPCR reactions were carried out on the 7500 Fast Real-Time PCR system (Applied Biosystems) using a SYBR Green PCR Master Mix (Applied Biosystems) and 2 ng cDNA per $10 \mu \mathrm{l}$ reactions. Three biological and two technical replicates of shoot samples were used for each condition. The relative expression of genes in infected plants compared to mockinoculated plants was calculated for each cultivar at each time point as ${ }_{\Delta \Delta} \mathrm{C}_{\mathrm{T}}$, based on the method described by Livak and Schmittgen (2001). Previously validated hop genes YLS8, DRH1 and CAC (Štajner et al. 2013) were used as references.

For comparison between RT-qPCR and RNA-Seq, $\log _{2} \mathrm{FC}$ values for differential expression between infected and control plants were calculated for the selected genes for each method separately and the results were then compared by calculating Pearson's product moment correlation coefficient $(r)$.

\section{Results}

\section{RNA sequencing and mapping}

In this study, we conducted a comprehensive time-course RNA-Seq experiment with infected and mock inoculated plants (Fig. 1) to uncover the global expression changes in shoot and root tissues of resistant and susceptible hop plants following $V$. nonalfalfae colonization.

High-throughput sequencing of 32 hop RNA samples yielded a total of $822.6 \mathrm{M}$ reads of length $50 \mathrm{bp}$, amounting to over $41 \mathrm{Gbp}$ of raw sequence data. At the trimming step, under $0.5 \%$ of reads were removed in each sample, so the final sequencing yield amounted to between 18.3 $\mathrm{M}$ and 38.3 $\mathrm{M}$ trimmed reads per sample (25.7 $\mathrm{M}$ on average). These were then mapped to the reference hop genome from HopBase (http://hopbase.cgrb.oregonstate. edu/) annotated with 46,735 gene models with a mean length of $2347 \mathrm{bp}$. Cytogenetic research and genome assembly analysis (Hill et al. 2017) revealed that the hop genome is highly repetitive, which causes difficulties in short-read genome assembly and transcript mapping. Based on the lack of a quality reference genome, the mapping rate was expected to be slightly lower. Of the trimmed reads, $49 \%$ mapped to exonic regions, $3 \%$ to intronic regions and $13 \%$ to intergenic regions, while $35 \%$ did not map to the reference genome. However, $65 \%$ of mapped reads is comparable with other RNA-Seq studies of hop: $70 \%$ for 'Shinsuwase' (Natsume et al. 2015) and $76 \%$ for 'Teamaker' (Hill et al. 2017) or other plants infected by Verticillium wilt-50\% mapped reads and 80-82\% mapped reads in two studies on cotton (Xu et al. 2011; Sun et al. 2013) and 71-89\% mapped reads in a study on tomato (Tan et al. 2015). The reads that mapped to exonic regions represented 181.5-fold average coverage of reference gene models. Of 46,735 annotated hop gene models, between 17,562 and 19,430 (37.6 and 41.6\%) were expressed $(\mathrm{CPM} \geq 1)$ in individual samples. Of all annotated gene models, 23,755 or $50.8 \%$ were expressed in at least one of the 32 conditions and were considered for further analysis to determine their differential expression.

\section{Differential expression of hop genes following infection with $V$. nonalfalfae}

Differential expression of genes in infected plants in comparison to control (mock inoculated) plants was determined for the four cultivar-tissue combinations (resistant shoots, susceptible shoots, resistant roots and 
susceptible roots) using FunPat, an $\mathrm{R}$ package developed for differential expression analysis in time-series experiments (Sanavia et al. 2015). We considered the genes with adjusted $p$ value less than 0.05 to be differentially expressed genes (DEGs). The complete list of DEGs for each cultivar-tissue combination, including differential expression data and annotations, is available in Online Resource 2 .

The total number of DEGs varied considerably among the cultivar-tissue combinations (Fig. 2a). There were fewer DEGs in roots than in shoots: in the susceptible cultivar, a total of 804 DEGs was determined in roots and 2259 DEGs in shoots, while in the resistant cultivar there were only 69 DEGs in roots and 4020 DEGs in shoots. In each cultivar-tissue combination, and especially in shoots of the resistant cultivar, more DEGs were down- than up-regulated. In shoots, there were 1056 DEGs common to both cultivars, 446 up-regulated and 610 down-regulated (Fig. 2b), while in roots only two up-regulated and one down-regulated DEGs were common to both cultivars.

\section{GO Slim and KEGG pathways enrichment of DEGs}

As a first step towards understanding the functional implications of hop transcriptional response to infection with $V$. nonalfalfae, DEGs were analysed for GO Slim Plant and KEGG enrichment in each cultivar-tissue combination. On average, $73 \%$ of DEGs per cultivar-tissue combination were associated with at least one GO Slim term, while $24 \%$ were associated with at least one KEGG pathway. The most enriched GO Slim biological process terms and the most enriched KEGG pathways are shown in Fig. 3. The enrichment analysis data for all other GO Slim terms and KEGG pathways are given in tabular format in Online Resource 3.

In the GO Slim category biological process (Fig. 3a), the most enriched terms were 'cellular process', to which 14-23\% of DEGs were assigned per cultivar-tissue combination, and 'biosynthetic process', with $12-15 \%$ of DEGs. The term 'carbohydrate metabolic process' was enriched with DEGs in roots of the susceptible cultivar (13\% of DEGs, compared to $6-8 \%$ in the other cultivartissue combinations). In roots of the resistant cultivar, $10 \%$ of DEGs were assigned to the term 'nucleobase-containing compound metabolic process' (compared to $3 \%$ in roots of the susceptible cultivar and $7-8 \%$ in shoots) and $6 \%$ of DEGs to 'transport' (compared to $3-4 \%$ in other combinations), while only $1 \%$ of DEGs were assigned to 'response to stress' (compared to $8 \%$ in roots of the susceptible cultivar and 5-6\% in shoots) and 3\% of DEGs to 'cellular component organization' (compared to $6 \%$ in other combinations).

The most enriched terms in the GO Slim category molecular function (Online Resource 3) were 'catalytic activity', to which 10-24\% of DEGs were assigned, 'binding', which represented 9-20\% of DEGs and 'hydrolase activity', which represented $9-17 \%$ of DEGs. The enrichment of all three terms was greatest in the susceptible cultivar, especially in roots, while they were less enriched in roots of the resistant cultivar. In contrast, the term ' $\mathrm{ki}$ nase activity' was most enriched in roots of the resistant cultivar (9\% of DEGs) and less in roots of the susceptible cultivar ( $3 \%$ of DEGs).
Fig. 2 The number of differentially expressed genes (DEGs) in hop plants infected with $V$. nonalfalfae based on pairwise comparison with their respective mock inoculated controls. a Numbers of up- and down-regulated DEGs per cultivar-tissue combination. b Venn diagram for number of DEGs in roots in common and specific to both cultivars. c Venn diagram for number of DEGs in shoots in common and specific to both cultivars. In $\mathbf{b}$ and $\mathbf{c}$ the number of upregulated genes is indicated above the horizontal line and the number of down-regulated genes below it

\section{a}

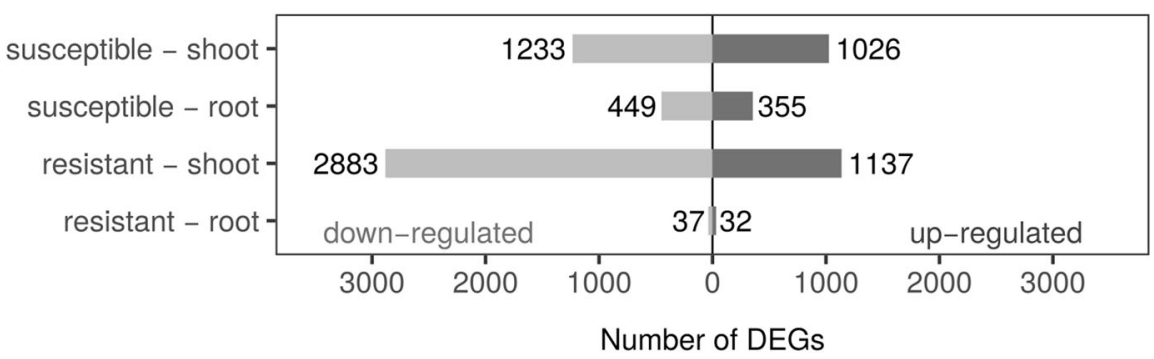

b

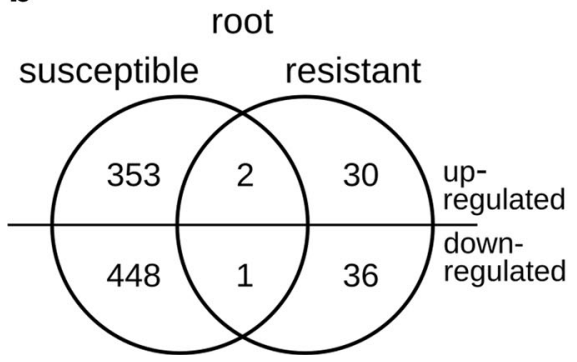

C

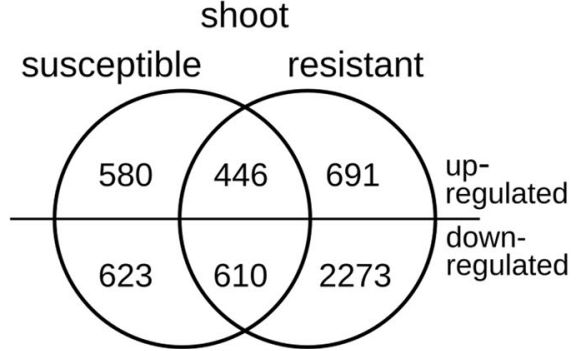



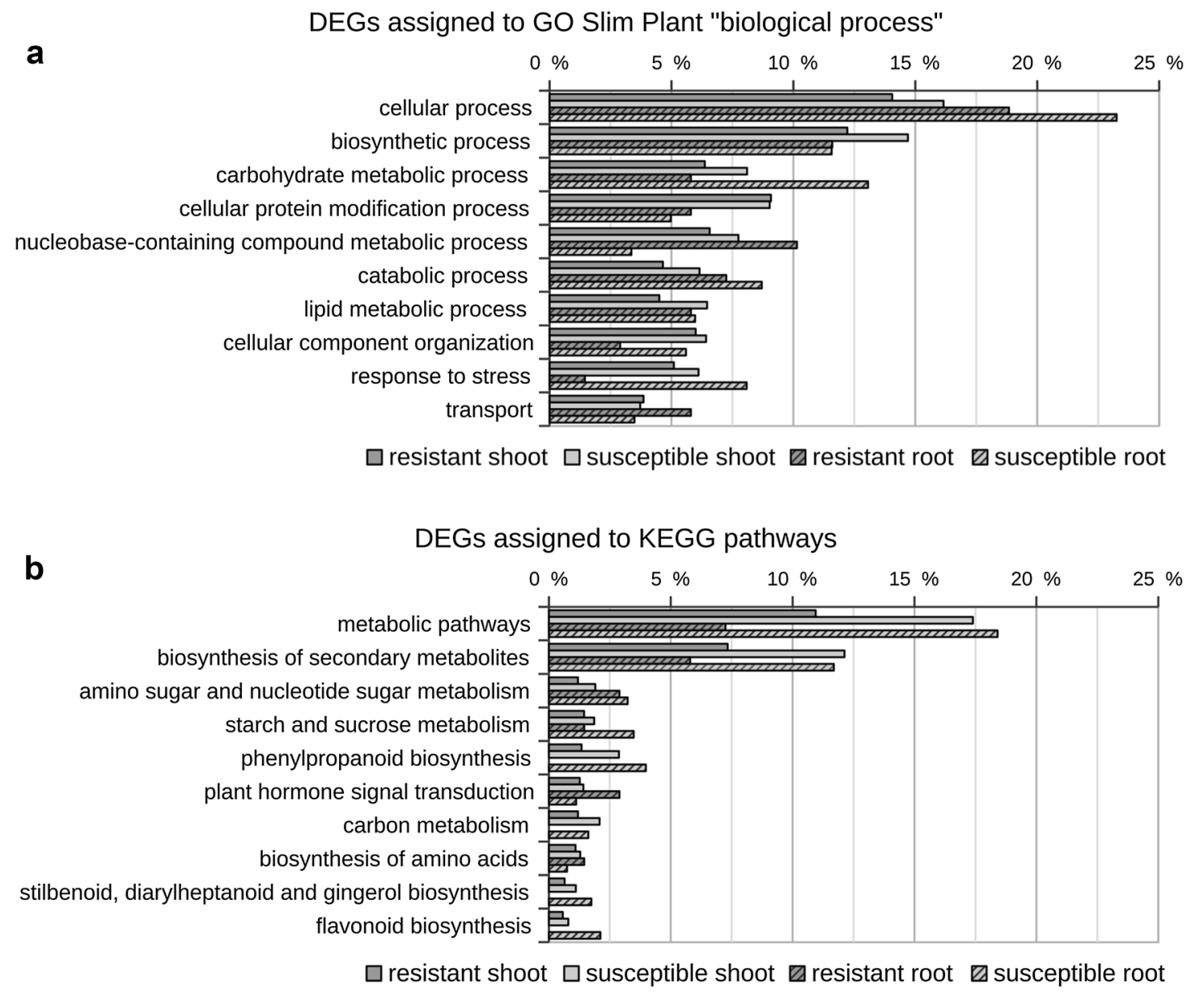

Fig. 3 GO Slim Plant term (a) and KEGG pathway (b) enrichment of differentially expressed hop genes following infection with $V$. nonalfalfae. The bars represent percentages of differentially

In GO Slim category cellular component (Online Resource 3), the most enriched terms were 'membrane' with 11-15\% of DEGs, 'plastid' with 4-14\% of DEGs and 'extracellular region', to which $3-8 \%$ of DEGs were assigned. The terms 'plastid' and 'thylakoid' were enriched in shoots of the susceptible cultivar (14 and 7\% of DEGs, respectively, compared to $4-7$ and $1-3 \%$ in other cultivartissue combinations), while 'extracellular region' and 'cell wall' were enriched in roots of the susceptible cultivar $(8$ and 5\% of DEGs, respectively, compared to $3-5$ and $1-2 \%$ in other combinations). In roots of the resistant cultivar, no DEGs were assigned to the terms 'plasma membrane' and 'cytosol', while in other cultivar-tissue combinations, these terms contained 3-4 and 2-3\% of DEGs, respectively.

In KEGG analysis (Fig. 3b), 18 and 17\% of DEGs in roots and shoots, respectively, of the susceptible cultivar were mapped to 'metabolic pathways' and 7 and $11 \%$ of DEGs in roots and shoots of the resistant cultivar. The 'biosynthesis of secondary metabolism' pathway was enriched in the susceptible cultivar (12\% of DEGs in both expressed genes (DEGs) assigned to an individual GO term or KEGG pathway relative to the total number of DEGs in a particular cultivar-tissue combination

roots and shoots compared to $6-7 \%$ in the resistant cultivar). Additionally, 'phenylpropanoid biosynthesis' was enriched in the susceptible cultivar (3-4\% of DEGs were mapped to the pathway, compared to $1 \%$ in shoots and no DEGs in roots of the resistant cultivar). Furthermore, in roots of the resistant cultivar, no DEGs were assigned to the pathways 'carbon metabolism', 'stilbenoid, diarylheptanoid and gingerol biosynthesis' and 'flavonoid biosynthesis'; however, in other combinations, only $1-2 \%$ of DEGs were mapped to these pathways. On the other hand, $3 \%$ of DEGs were assigned to the pathway 'plant hormone signal transduction' in roots of the resistant cultivars, compared to $1 \%$ for other combinations.

\section{Network-based analysis of functional enrichment}

To gain further and more specific insight into the processes in which DEGs are involved, we performed network-based analysis of Gene Ontology enrichment for the top 100 DEGs from each cultivar-tissue combination using 
Cytoscape plugin ClueGO (Bindea et al. 2009). The results of the analysis for each cultivar-tissue combination are shown in Table 1.

In the susceptible cultivar, the enrichment of GO terms with DEGs was similar between shoots and roots. The majority of enriched GO terms were associated with defence response and included terms such as 'response to wounding', 'response to jasmonic acid', 'defence response, incompatible interaction' and 'chitinase activity' in both roots and shoots and also 'innate immune response' and 'systemic acquired resistance' in shoots. Interestingly, none of these terms were found to be enriched in the resistant cultivar. Unique to the roots of the susceptible cultivar was a group of GO terms associated with ribonuclease activity, while enrichment of a group containing photosynthesisrelated GO terms was specific to the shoots of the susceptible cultivar (Table 1).

Functional enrichment in the resistant cultivar was different between roots and shoots and to that in the susceptible cultivar. In roots, the GO term 'polysaccharide catabolic process' and a group associated with terpenoid biosynthesis were enriched with DEGs, while the most significantly enriched GO terms in shoots were "cutin biosynthetic process' and a group containing GO terms associated with (secondary) cell wall biogenesis, followed by a group involved in dicarboxylic acid metabolism, a group related to lateral root development and GO cellular compartment terms associated with membrane, cell wall and vacuole (Table 1).

\section{Significant temporal profiles of differentially expressed genes}

STEM analysis (Ernst and Bar-Joseph 2006) was used to identify coordinate differential expression of hop genes during the time course of $V$. nonalfalfae colonization. Among the top 100 DEGs analysed for each cultivar-tissue combination, nine significant temporal profiles $\left(p_{\text {adj. }}<0.05\right)$ were identified: 2 and 4 profiles in shoots and roots, respectively, of the susceptible cultivar and 3 in shoots of the resistant cultivar (Online Resource 5), while there were no significant time-course profiles in roots of the resistant cultivar. The DEGs belonging to each of the significant temporal profiles and the enrichment of individual profiles with GO terms are listed in Online Resource 6.

Figure 4 shows the most significant temporal profiles of DEGs in shoots and roots of the susceptible cultivar and in shoots of the resistant cultivar. The profile in shoots of the susceptible cultivar, to which 45 out of 100 DEGs in that cultivar-tissue combination were mapped, was characterized by a constantly increasing up-regulation during the course of the experiment (Fig. 4a). Although there were no significantly enriched GO terms for this profile in this cultivar-tissue combination, some GO terms were associated with more than half of the DEGs in this temporal profile, such as 'response to stress' and 'response to biotic stimulus' (Online Resource 6). The same temporal profile was significant in roots of the susceptible cultivar, in which 21 of 100 DEGs were mapped to it $\left(p_{\text {adj. }}=1.7 \times 10^{-14}\right.$; Online Resource 5). In this cultivar-tissue combination, 9 GO terms were significantly enriched $\left(p_{\text {adj. }}<0.05\right)$ and included 'innate immune response' and 'defence response to fungus, incompatible interaction' (Online Resource 6).

However, the most significant temporal profile in roots of the susceptible cultivar, to which 34 of 100 DEGs were mapped, encompassed both up- and down-regulation: starting with little differential regulation at $6 \mathrm{dpi}$, it exhibited up-regulation at $12 \mathrm{dpi}$ and then increasing down-regulation at 18 and $30 \mathrm{dpi}$ (Fig. 4b). Two GO terms were significantly enriched for this profile: 'lipid metabolic process' with 11 associated DEGs and 'small molecule metabolic process' with 10 DEGs (Online Resource 6). This temporal profile was also significant in shoots of the susceptible cultivar $\left(p_{\text {adj. }}=1.6 \times 10^{-8}\right)$ and included 15 out of 100 DEGs (Online Resource 5). The assortment of GO terms associated with this profile was similar to that in roots (Online Resource 6), but none were significantly enriched.

The most significant temporal profile in shoots of the resistant cultivar, which included 28 out of 100 DEGs, showed consistent down-regulation, which was especially pronounced at $18 \mathrm{dpi}$ (Fig. 4c). It was not significantly enriched with any GO term but it included, for example, 9 DEGs associated with 'lipid metabolic process', 6 associated with 'defence response' and 5 associated with 'cell wall organization or biogenesis' (Online Resource 6).

There were four more significant temporal profiles: two in roots of the susceptible cultivar, with 10 and 8 out of 100 DEGs and with a general upward tendency, while a further two were in shoots of the resistant cultivar, with 15 and 10 out of 100 DEGs and with a general downward tendency (Online Resource 5). None of them were significantly enriched with any GO terms (Online Resource 6).

\section{Validation of RNA-Seq expression patterns with quantitative reverse transcription polymerase chain reaction}

To validate the reliability of expression patterns obtained by RNA-Seq, we performed quantitative reverse transcription polymerase chain reaction (RT-qPCR) on twelve selected DEGs in shoots of both cultivars using three biological replicates for each condition. The genes were selected from among those that were determined by FunPat as differentially expressed in at least one of the cultivar- 
Table 1 The results of network-based analysis of functional enrichment for GO terms associated with DEGs in hop after $V$. nonalfalfae infection

\begin{tabular}{|c|c|c|c|c|c|c|c|}
\hline \multirow[t]{2}{*}{ GO ID } & \multicolumn{3}{|l|}{ Susceptible—shoot } & \multirow[t]{2}{*}{ GO ID } & \multicolumn{3}{|l|}{ Resistant-shoot } \\
\hline & GO Term & $\mathbf{p}[$ term $]$ & $\mathbf{p}$ [group] & & GO Term & $\mathbf{p}[$ term $]$ & $\mathbf{p}$ [group] \\
\hline GO:0016701 & $\begin{array}{l}\text { MF_oxidoreductase activity, acting } \\
\text { on... }\end{array}$ & $1.7 \mathrm{e}-4$ & $9.2 \mathrm{e}-5$ & GO:0010143 & $\mathrm{BP}$-cutin biosynthetic process & $7.3 e-4$ & $3.6 \mathrm{e}-4$ \\
\hline GO:0080027 & $\mathrm{BP}$-response to herbivore & $3.5 \mathrm{e}-6$ & $2.5 \mathrm{e}-4$ & GO:0009834 & $\begin{array}{l}\text { BP-plant-type secondary cell wall } \\
\text { biogenesis }\end{array}$ & $7.4 \mathrm{e}-4$ & $4.1 \mathrm{e}-3$ \\
\hline GO:0031408 & $\mathrm{BP}$-oxylipin biosynthetic process & $1.8 \mathrm{e}-5$ & & GO:0042546 & $\mathrm{BP}$-cell wall biogenesis & $9.0 \mathrm{e}-3$ & \\
\hline GO:0031407 & $\mathrm{BP} \_$oxylipin metabolic process & $1.8 \mathrm{e}-5$ & & GO:0009832 & BP-plant-type cell wall biogenesis & $1.4 \mathrm{e}-2$ & \\
\hline GO:0009611 & $\mathrm{BP}$-response to wounding & $6.6 \mathrm{e}-4$ & & GO:0016830 & MF-carbon-carbon lyase activity & $8.5 \mathrm{e}-3$ & $8.2 \mathrm{e}-3$ \\
\hline GO:0009753 & $\mathrm{BP}$-response to jasmonic acid & $1.0 \mathrm{e}-3$ & & GO:0016831 & MF-carboxy-lyase activity & $2.4 \mathrm{e}-2$ & \\
\hline GO:0006633 & $\mathrm{BP}$-fatty acid biosynthetic process & $1.6 \mathrm{e}-2$ & & GO:0016903 & $\begin{array}{l}\text { MF—oxidoreductase activity, acting } \\
\text { on... }\end{array}$ & $2.4 \mathrm{e}-2$ & \\
\hline GO:0008061 & MF_chitin binding & $1.6 \mathrm{e}-4$ & $4.0 \mathrm{e}-4$ & GO:0043648 & $\begin{array}{l}\text { BP_dicarboxylic acid metabolic } \\
\text { process }\end{array}$ & $2.5 \mathrm{e}-2$ & \\
\hline GO:0004568 & MF_chitinase activity & $6.2 \mathrm{e}-4$ & & GO:0010102 & $\mathrm{BP}$-lateral root morphogenesis & $1.0 \mathrm{e}-2$ & $1.0 \mathrm{e}-2$ \\
\hline GO:0045087 & $\mathrm{BP}$-innate immune response & $9.1 \mathrm{e}-4$ & $4.5 \mathrm{e}-4$ & GO:0010101 & $\begin{array}{l}\mathrm{BP} \text {-post-embryonic root } \\
\text { morphogenesis }\end{array}$ & $1.0 \mathrm{e}-2$ & \\
\hline GO:0009814 & $\begin{array}{l}\mathrm{BP} \text {-defence response, incompatible } \\
\text { interaction }\end{array}$ & $2.4 \mathrm{e}-3$ & & GO:0048528 & $\begin{array}{l}\text { BP-post-embryonic root } \\
\text { development }\end{array}$ & $1.5 \mathrm{e}-2$ & \\
\hline GO:0009627 & $\mathrm{BP}-$ systemic acquired resistance & $1.3 \mathrm{e}-2$ & & GO:0048527 & $\mathrm{BP}$-lateral root development & $2.0 \mathrm{e}-2$ & \\
\hline GO:0016835 & MF_carbon-oxygen lyase activity & $1.2 \mathrm{e}-2$ & $1.2 \mathrm{e}-2$ & GO:0048645 & $\mathrm{BP}$ - organ formation & $2.2 \mathrm{e}-2$ & \\
\hline GO:0052689 & $\begin{array}{l}\text { MF_carboxylic ester hydrolase } \\
\text { activity }\end{array}$ & $2.1 \mathrm{e}-2$ & $1.5 \mathrm{e}-2$ & GO:0009886 & $\begin{array}{l}\mathrm{BP} \text {-post-embryonic } \\
\text { morphogenesis }\end{array}$ & $2.3 e-2$ & \\
\hline GO:0009642 & $\mathrm{BP}-$ response to light intensity & $2.4 \mathrm{e}-2$ & $1.6 e-2$ & GO:0031225 & $\begin{array}{l}\mathrm{CC} \text {-anchored component of } \\
\text { membrane }\end{array}$ & $2.2 \mathrm{e}-2$ & $1.1 \mathrm{e}-2$ \\
\hline GO:0010287 & $\mathrm{CC}$-plastoglobule & $9.4 \mathrm{e}-4$ & $1.7 \mathrm{e}-2$ & GO:0009505 & $\mathrm{CC}$-plant-type cell wall & $2.4 \mathrm{e}-2$ & $1.2 \mathrm{e}-2$ \\
\hline GO:0009523 & CC-photosystem II & $1.4 \mathrm{e}-2$ & & GO:0000325 & $\mathrm{CC}$-plant-type vacuole & $2.0 \mathrm{e}-2$ & $1.3 \mathrm{e}-2$ \\
\hline GO:0019684 & $\mathrm{BP}$-photosynthesis, light reaction & $1.7 \mathrm{e}-2$ & & & & & \\
\hline GO:0009521 & $\mathrm{CC}$-photosystem & $2.0 \mathrm{e}-2$ & & & & & \\
\hline \multirow[t]{2}{*}{ GO ID } & \multicolumn{3}{|l|}{ Susceptible-root } & \multirow[t]{2}{*}{ GO ID } & \multicolumn{3}{|l|}{ Resistant-root } \\
\hline & GO Term & $\mathbf{p}[$ term $]$ & $\mathbf{p}$ [group] & & GO Term & $\mathbf{p}[$ term $]$ & $\mathbf{p}$ [group] \\
\hline GO:0080027 & $\mathrm{BP}$-response to herbivore & $1.5 \mathrm{e}-6$ & $7.9 \mathrm{e}-7$ & GO:0000272 & $\begin{array}{l}\mathrm{BP} \text { - polysaccharide catabolic } \\
\text { process }\end{array}$ & $1.4 \mathrm{e}-2$ & $9.3 e-3$ \\
\hline GO:0009611 & $\mathrm{BP}$-response to wounding & $2.0 \mathrm{e}-5$ & $1.7 \mathrm{e}-4$ & GO:0010333 & MF-terpene synthase activity & $1.8 \mathrm{e}-4$ & $8.7 \mathrm{e}-2$ \\
\hline GO:0009753 & $\mathrm{BP}$-response to jasmonic acid & $3.4 \mathrm{e}-5$ & & GO:0016838 & $\begin{array}{l}\text { MF—carbon-oxygen lyase activity, } \\
\text { acting on... }\end{array}$ & $2.4 \mathrm{e}-4$ & \\
\hline GO:0031408 & $\mathrm{BP}$-oxylipin biosynthetic process & $4.0 \mathrm{e}-4$ & & GO:0016835 & MF_carbon-oxygen lyase activity & $1.0 \mathrm{e}-2$ & \\
\hline GO:0031407 & $\mathrm{BP}$-oxylipin metabolic process & $4.0 \mathrm{e}-4$ & & GO:0000287 & MF_-magnesium ion binding & $1.0 \mathrm{e}-2$ & \\
\hline GO:0051213 & MF_dioxygenase activity & $1.0 \mathrm{e}-2$ & & GO:0016114 & $\mathrm{BP}$-terpenoid biosynthetic process & $1.8 \mathrm{e}-2$ & \\
\hline GO:0008061 & MF_chitin binding & $7.6 e-5$ & $2.2 \mathrm{e}-4$ & & & & \\
\hline GO:0004568 & MF_chitinase activity & $2.9 \mathrm{e}-4$ & & & & & \\
\hline GO:0009814 & $\begin{array}{l}\mathrm{BP} \text {-defence response, incompatible } \\
\text { interaction }\end{array}$ & $1.1 \mathrm{e}-2$ & $6.3 e-3$ & & & & \\
\hline GO:0048528 & $\begin{array}{l}\text { BP-post-embryonic root } \\
\text { development }\end{array}$ & $1.6 \mathrm{e}-2$ & $1.1 \mathrm{e}-2$ & & & & \\
\hline GO:0004540 & MF_ribonuclease activity & $7.8 \mathrm{e}-3$ & $1.3 \mathrm{e}-2$ & & & & \\
\hline GO:0090305 & $\begin{array}{l}\mathrm{BP} — \text { nucleic acid phosphodiester } \\
\text { bond hydrolysis }\end{array}$ & $9.2 \mathrm{e}-3$ & & & & & \\
\hline
\end{tabular}


Table 1 continued

\begin{tabular}{|c|c|c|c|c|c|c|c|}
\hline \multirow[t]{2}{*}{ GO ID } & \multicolumn{3}{|l|}{ Susceptible-root } & \multirow[t]{2}{*}{ GO ID } & \multicolumn{3}{|c|}{ Resistant-root } \\
\hline & GO Term & $\mathbf{p}[$ term $]$ & $\mathbf{p}$ [group] & & GO Term & $\mathbf{p}[$ term] & $\mathbf{p}$ [group] \\
\hline GO:0090501 & $\begin{array}{l}\text { BP-RNA phosphodiester bond } \\
\text { hydrolysis }\end{array}$ & $1.0 \mathrm{e}-2$ & & & & & \\
\hline GO:0004518 & $\mathrm{MF}$ —nuclease activity & $1.6 \mathrm{e}-2$ & & & & & \\
\hline GO:0000325 & $\mathrm{CC}$-plant-type vacuole & $1.9 \mathrm{e}-2$ & $1.4 \mathrm{e}-2$ & & & & \\
\hline
\end{tabular}
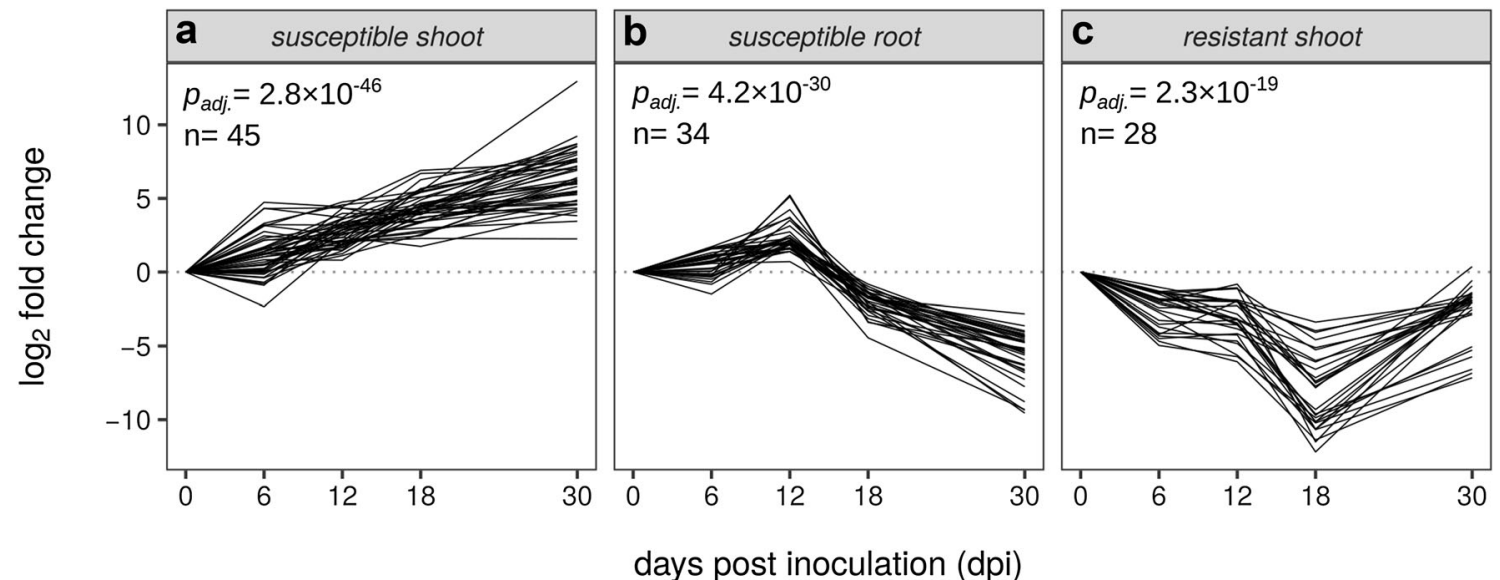

Fig. 4 The most significant temporal differential expression profiles of the top 100 DEGs in shoots of the susceptible hop (a), in roots of the susceptible hop (b) and in shoots of the resistant hop (c) after $V$.

tissue combinations and so that half of them were predominantly up- and half predominantly down-regulated (Table 2). The results of RT-qPCR analysis for the selected genes are shown in Fig. 5a and are also available in tabular format in Online Resource 4.

To evaluate the correlation between the two methods statistically, we calculated Pearson's product moment correlation coefficient $(r)$ between the $\log _{2} \mathrm{FC}$ values obtained by RNA-Seq and the average $\log _{2} \mathrm{FCs}$ obtained by RT-qPCR (Fig. 5b). The correlation coefficient was 0.86 with a $p$ value $<2.2 \times 10^{-16}$ and $95 \%$ confidence interval between 0.80 and 0.90 , indicating a significant and strong correlation between the methods, thus validating the results obtained by RNA-Seq on which the functional enrichment analyses were performed.

\section{Discussion}

This study is the first large-scale transcriptomic RNA-Seq analysis of hop response to infection with Verticillium nonalfalfae, the main causative agent of Verticillium wilt in hops. In an extensive time-course survey, the nonalfalfae infection. For each profile, the number of assigned DEGs out of the top 100 for the corresponding cultivar-tissue combination and their adjusted $p$ values are given

transcriptional responses in roots and shoots of both resistant and susceptible cultivars were determined in infected and healthy plants. The expression levels were monitored at $6,12,18$ and 30 dpi to match the colonization dynamics of $V$. nonalfalfae in hop, from initial colonization to full symptom development in the susceptible cultivar between 20 and 30 dpi (Cregeen et al. 2015; Flajšman et al. 2017). After root penetration and initial spread of the fungus in the roots of the resistant cultivar, a decline in fungal biomass is observed around 20 dpi due to plant resistant responses and further growth in hop stem is very restricted. Similar systemic colonization in susceptible plants, and restriction of the pathogen to the roots and stem base of resistant plants, has also been reported for $V$. longisporum in oilseed rape (Eynck et al. 2009) and for $V$. dahliae in olives (Markakis et al. 2010).

In the present study, a large amount of expression data was generated during the experiment and processed with a state of the art bioinformatics pipeline to gain insight into functional enrichment of biological processes with differentially expressed genes. The most prominent difference between the cultivars was that the susceptible plants exhibited a much stronger general defence response than 
Table 2 Candidate hop genes expressed in V. nonalfalfae-interactions and their best Arabidopsis blastx matches

\begin{tabular}{llll}
\hline HopBase ID & Expression trend & Best TAIR hit & \\
\hline$H L . S W . v 1.0 . G 035683$ & $\mathrm{Up}$ & $A T 4 G 33720$ & CAP superfamily protein \\
$H L . S W \cdot v 1.0 . G 023951$ & $\mathrm{Up}$ & $A T 5 G 43580$ & Unusual serine protease inhibitor \\
$H L . S W \cdot v 1.0 . G 036575$ & $\mathrm{Up}$ & $A T 2 G 38540$ & Lipid transfer protein 1 \\
$H L . S W \cdot v 1.0 . G 030451$ & $\mathrm{Up}$ & $A T 5 G 24080$ & Protein kinase superfamily protein \\
$H L . S W . v 1.0 . G 015232$ & $\mathrm{Up}$ & $A T 3 G 50980$ & Dehydrin xero 1 \\
$H L . S W . v 1.0 . G 030241$ & $\mathrm{Up}$ & $A T 2 G 45220$ & Pectin methylesterase 17 \\
$H L . S W \cdot v 1.0 . G 017076$ & Down & $A T 1 G 24020$ & MLP-like protein 423 \\
$H L . S W \cdot v 1.0 . G 039151$ & Down & $A T 5 G 15310$ & MYB domain protein 16 \\
$H L . S W . v 1.0 . G 015902$ & Down & $A T 5 G 45950$ & GDSL esterase/acyltransferase/lipase \\
$H L . S W . v 1.0 . G 007127$ & Down & $A T 5 G 33370$ & GDSL-like lipase/acylhydrolase superfamily protein \\
$H L . S W . v 1.0 . G 009568$ & Down & $A T 2 G 45970$ & CYP86A8, lacerata \\
$H L . S W . v 1.0 . G 018736$ & Down & $A T 1 G 49430$ & Long-chain acyl-CoA synthetase 2 \\
\hline
\end{tabular}

the resistant ones, and the cultivars also exhibited differences in response of genes involved in the jasmonate pathway and regulation of photosynthesis, as well as terpenoid biosynthesis and cell wall metabolism.

Differentially expressed genes (DEGs) were determined with FunPat (Sanavia et al. 2015) for four separate cultivartissue combinations (shoots and roots of the resistant and the susceptible hop) by comparing the temporal profiles of gene expression in infected plants with those in the control (mock inoculated). Validation of the expression patterns of DEGs with RT-qPCR on twelve selected DEGs resulted in a significant correlation between the methods, with Pearson's correlation coefficient of $r=0.86$, which is comparable to transcriptomic study of cotton- $V$. dahliae interaction ( $\mathrm{Xu}$ et al. 2011). In the roots of the resistant cultivar, there were far fewer DEGs than in the roots of the susceptible cultivar. This is consistent with the results of a proteomic study on the same pathosystem (Mandelc et al. 2013), in which 252 infection-specific protein spots were discovered in the susceptible hop and no changes in the resistant cultivar after infection with $V$. nonalfalfae, possibly indicating the involvement of constitutive rather than induced defence mechanisms in hop resistance to the fungus. A less intense response in roots of the resistant compared to the susceptible cultivar is also reported in transcriptomic studies of Verticillium wilt on other hosts. In a study of tomato infected by Verticillium dahliae (van Esse et al. 2009), the microarray results indicated a similar disproportion between the number of DEGs in roots of resistant and susceptible plants at 3 and $7 \mathrm{dpi}$ : there were 147 DEGs in roots of the resistant compared to 1188 DEGs in roots of the susceptible tomato plants. Similarly, in Medicago truncatula infected by Verticillium alfalfae (Toueni et al. 2016), the response in roots of a susceptible cultivar was stronger, with 4053 detected DEGs, compared to 1055 DEGs detected in roots of a resistant cultivar.
These findings suggest that susceptibility to Verticillium infection is not due to lack of immune responses, but is rather caused by some failure in the efficacy of the responses.

Enrichment analysis of plant biologic and metabolic processes with GO Slim and KEGG pathways revealed a higher percentage of DEGs in the roots of the susceptible hop associated with 'carbohydrate metabolic process' and 'hydrolase activity', 'biosynthesis of secondary metabolites', 'phenylpropanoid biosynthesis' and 'response to stress'. On the other hand, in the roots of the resistant hop, a higher percentage of DEGs were attributed to 'nucleobase-containing compound metabolic process', 'transport', 'kinase activity' and 'plant hormone signal transduction'. In contrast to our results, previous transcriptome studies of the $V$. dahliae interaction with cotton (Xu et al. 2011) and with tomato (Gayoso et al. 2010) detected up-regulation of phenylpropanoid metabolism and synthesis of lignins as prominent processes contributing to a successful defence response of resistant plants. The differences could be attributed to a different mechanism of resistance in those pathosystems, especially since resistance in tomato is mediated by $\mathrm{Ve} 1$ receptor-like protein (Fradin et al. 2009; Gayoso et al. 2010).

A more profound insight into the functional implications of hop response to $V$. nonalfalfae was provided using STEM (Ernst and Bar-Joseph 2006) and network-based analysis of functional enrichment by ClueGo (Bindea et al. 2009). Interestingly, a significant temporal profile, with increasing gene expression of PR-4 homologs and thaumatin, was detected only in the susceptible hop and corroborated by network-based enrichment of GO terms such as 'innate immune response', 'defence response, incompatible interaction', 'response to stress' and 'chitinase activity'. These results are consistent with previous studies of the hop- $V$. nonalfalfae interaction on both proteomic 

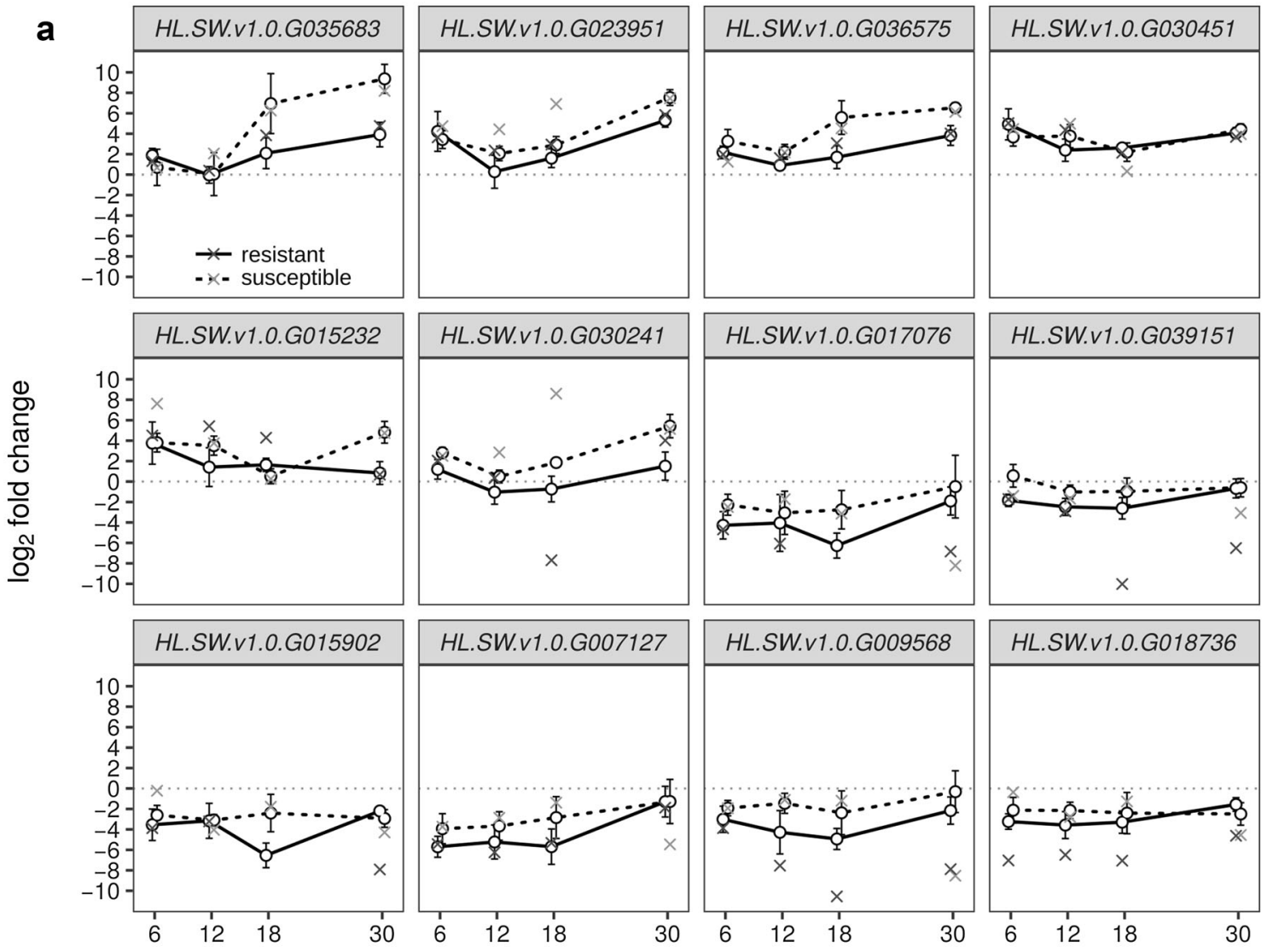

days post inoculation (dpi)

b

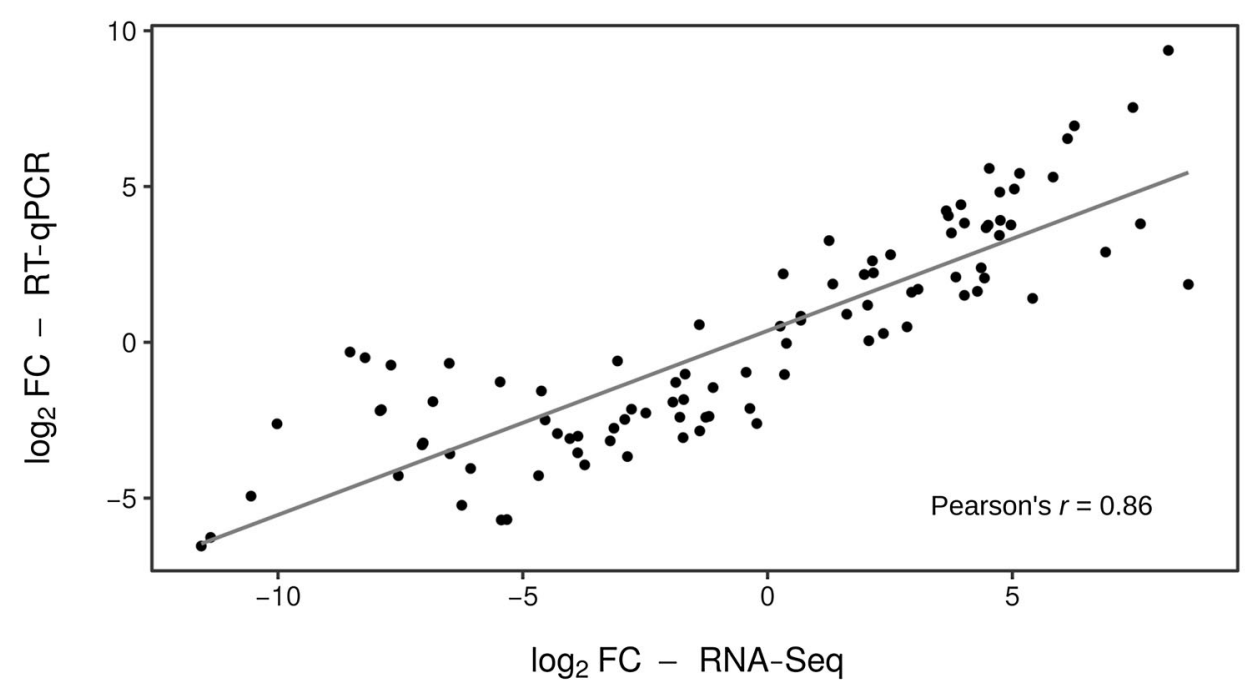

Fig. 5 Reverse transcription quantitative polymerase chain reaction (RT-qPCR) analysis of selected hop genes differentially expressed during $V$. nonalfalfae infection and comparison with RNA-Seq results. a Plots represent $\log _{2}$ fold changes between expression in infected and mock-inoculated plants determined with RT-qPCR and using previously validated reference genes Yellow leaf specific protein $8, Y L S 8$, DEAD box RNA helicase, DRH1, and Clathrin adaptor complexes medium subunit, $C A C$ (Štajner et al. 2013). Errorbars indicate standard error $(n=3)$. The crosses represent $\log _{2} \mathrm{FC}$ values as determined by RNA-Seq. b Comparison of $\log _{2} \mathrm{FC}$ determined by RNA-Seq and RT-qPCR using Pearson's product moment correlation coefficient. The points represent individual measurements of the same sample by the two methods and a trend line is added 
(Mandelc et al. 2013) and transcriptomic levels (Cregeen et al. 2015), showing an accumulation of defence-related proteins (such as chitinases, $\beta$-glucanases and thaumatinlike proteins) and steadily increasing expression of genes for pathogenesis-related proteins only in the susceptible hop. Intensive up-regulation of defence-related genes in susceptible but not in resistant plants was also observed in tomato following infection with $V$. dahliae (Robb et al. 2012). Taken together, these findings indicate that a substantial general defence response is mounted in the susceptible plants attacked by Verticillium and it intensifies during the time-course of colonization but is ultimately ineffective in protecting the plant from the fungus.

We identified another group of significantly enriched processes in both shoots and roots of the susceptible cultivar, which included GO terms such as 'response to jasmonic acid' and 'oxylipin metabolic process', and found a corresponding significant temporal profile with peak of gene expression at $12 \mathrm{dpi}$. DEGs in this profile were, for example, homologs of lipoxygenase 2 and allene oxide synthase, which are also important members of the jasmonic acid biosynthesis pathway (Wasternack and Song 2016). These results suggest a potential involvement of jasmonic acid signalling in the hop interaction with $V$. nonalfalfae. In addition, it has been shown that $V$. longisporum interferes with the jasmonic acid signalling pathway in Arabidopsis thaliana by direct interaction with jasmonic acid receptor COI1, thus enhancing the susceptibility of the host (Ralhan et al. 2012). Similar jasmonic acid signalling interference was reported in the interaction between $A$. thaliana and Fusarium oxysporum (Thatcher et al. 2009).

GO term enrichment of processes related to photosynthesis was also of interest. Consistent with global downregulation of photosynthesis in lettuce in response to $V$. dahliae colonization (Klosterman et al. 2011) and other studies of plant-pathogen interactions (Bilgin et al. 2010), several DEGs associated with photosynthesis (e.g., chlorophyll A/B binding protein 1 and photosystem I light harvesting complex gene 2) were down-regulated in shoots of the susceptible hop.

In roots of the resistant cultivar, we found no significant temporal expression profiles and detected only two enriched processes, polysaccharide catabolic process and terpenoid biosynthesis. In addition to antibacterial and antifungal properties of root-secreted terpenoid compounds (Baetz and Martinoia 2014), a strong production of terpenoids, especially in roots of a resistant cultivar, has also been reported in cotton plants infected with $V$. dahliae (Daayf et al. 1997) and in roots of tomato seedlings infected with V. albo-atrum (Hutson and Smith 1983). On the other hand, certain monoterpenes can stimulate germination of $V$. longisporum, and thus actually enhance infection of Arabidopsis roots (Roos et al. 2015).
In shoots of the resistant cultivar, according to networkbased functional analysis, various processes were enriched, including modification of cell wall and biosynthesis of cutin. A significant temporal profile, with the strongest gene down-regulation at $18 \mathrm{dpi}$, was linked to these processes and among others comprised gene homologs of cell wall associated pectinesterases and fasciclin-like proteins, as well as homologs of cutin metabolism associated GDSL lipases and long-chain acyl-CoA synthetase. In addition to serving as a preformed physical barrier, the cell wall responds dynamically to biotic stress by the formation of lignin and suberin, depositions of callose and cross-linking and remodelling of the cell wall structure (Bellincampi et al. 2014). It undergoes significant modifications also due to pathogen released cell wall-degrading enzymes, which have been clearly associated with pathogenicity of $V$. dahliae (Gharbi et al. 2015). Cutin metabolism was the most significantly enriched GO process in resistant hop. Cutin is polyesters of mostly oxygenated fatty acids and a major component of the plant cuticle (Fich et al. 2016). Although the disruption of cutin metabolism in A. thaliana increased cuticle permeability and led to strong resistance against necrotrophic fungus Botrytis cinerea (Bessire et al. 2007), we did not expect a correlation between the vascular pathogen $V$. nonalfalfae and resistance response related to the cuticle.

In conclusion, this study is the first transcriptome-wide RNA-Seq study of resistant and susceptible hop response to infection with $V$. nonalfalfae, which causes a devastating disease hampering the production of hop. The results suggest a strong general defence response in both shoots and roots of the susceptible cultivar, which is in accordance with the findings of previous studies on the same pathosystem, as well as of studies of Verticillium wilt on some other hosts. A vast amount of expression data was generated and processed in this study, providing a global and multifaceted overview of hop response to $V$. nonalfalfae, encompassing both susceptible and resistant plant responses in shoots and roots during the course of colonization. It is thus a useful addition to research of hop resistance to $V$. nonalfalfae and will contribute to eventual identification of its underlying mechanisms, with potential use in hop breeding programmes in the future.

Author contribution statement BJ conceived and coordinated the study and BJ, SB, NŠ and JJ participated in its design. SR prepared the plant and fungal material and performed hop inoculations. VP harvested and processed the samples. VP performed RT-qPCR analyses together with NS and bioinformatics analysis together with JJ. SB, VP and BJ analysed and interpreted the data and wrote the manuscript. All authors read and approved the manuscript. 
Acknowledgements The authors would like to thank Dr Slavko Mandelc for his contribution at the earliest stages of the study and Dr Federica Cattonaro from IGA Technologies for help with NGS sequencing.

\section{Compliance with ethical standards}

Funding The research was funded by the Slovenian Research Agency through Grant P4-0077 and student Grant 1000-13-0510 to VP.

Conflict of interest The authors declare that they have no conflict of interest.

Open Access This article is distributed under the terms of the Creative Commons Attribution 4.0 International License (http://crea tivecommons.org/licenses/by/4.0/), which permits unrestricted use, distribution, and reproduction in any medium, provided you give appropriate credit to the original author(s) and the source, provide a link to the Creative Commons license, and indicate if changes were made.

\section{References}

Altschul SF, Madden TL, Schäffer AA, Zhang J, Zhang Z, Miller W, Lipman DJ (1997) Gapped BLAST and PSI-BLAST: a new generation of protein database search programs. Nucleic Acids Res 25:3389-3402

Baetz U, Martinoia E (2014) Root exudates: the hidden part of plant defense. Trends Plant Sci 19:90-98

Bellincampi D, Cervone F, Lionetti V (2014) Plant cell wall dynamics and wall-related susceptibility in plant-pathogen interactions. Front Plant Sci 5:228. doi:10.3389/fpls.2014.00228

Bessire M, Chassot C, Jacquat A-C, Humphry M, Borel S, Petetot JMC, Metraux J-P, Nawrath C (2007) A permeable cuticle in Arabidopsis leads to a strong resistance to Botrytis cinerea. EMBO J 26:2158-2168

Bilgin DD, Zavala JA, Zhu J, Clough SJ, Ort DR, Delucia EH (2010) Biotic stress globally downregulates photosynthesis genes. Plant Cell Environ 33:1597-1613. doi:10.1111/j.1365-3040.2010. 02167.x

Bindea G, Mlecnik B, Hackl H, Charoentong $\mathrm{P}$, Tosolini M, Kirilovsky A, Fridman W-H, Pagès F, Trajanoski Z, Galon J (2009) ClueGO: a Cytoscape plug-into decipher functionally grouped gene ontology and pathway annotation networks. Bioinformatics 25:1091-1093. doi:10.1093/bioinformatics/ btp101

Chen YC, Wong CL, Muzzi F, Vlaardingerbroek I, Kidd BN, Schenk PM (2014) Root defense analysis against Fusarium oxysporum reveals new regulators to confer resistance. Sci Rep 4:5584

Conesa A, Gotz S, Garcia-Gomez JM, Terol J, Talon M, Robles M (2005) Blast2GO: a universal tool for annotation, visualization and analysis in functional genomics research. Bioinformatics 21:3674-3676

Cregeen S, Radišek S, Mandelc S, Turk B, Štajner N, Jakše J, Javornik B (2015) Different gene expressions of resistant and susceptible hop cultivars in response to infection with a highly aggressive strain of Verticillium albo-atrum. Plant Mol Biol Rep 33:689-704

Crowe F, Parks R (2001) Central Oregon agricultural research center 2000 annual report: fungicide screening for control of Verticillium wilt of peppermint. Central Oregon Agricultural Research Center, Oregon State University, Oregon
Daayf F, Nicole M, Boher B, Pando A, Geiger JP (1997) Early vascular defense reactions of cotton roots infected with a defoliating mutant strain of Verticillium dahliae. Eur J Plant Pathol 103:125-136. doi:10.1023/a:1008620410471

De Coninck B, Timmermans P, Vos C, Cammue BP, Kazan K (2014) What lies beneath: belowground defense strategies in plants. Trends Plant Sci 20:91-101

de Jonge R, van Esse HP, Maruthachalam K, Bolton MD, Santhanam P, Saber MK, Zhang Z, Usami T, Lievens B, Subbarao KV et al (2012) Tomato immune receptor Ve1 recognizes effector of multiple fungal pathogens uncovered by genome and RNA sequencing. Proc Natl Acad Sci 109:5110-5115

Di Camillo B, Toffolo G, Nair SK, Greenlund LJ, Cobelli C (2007) Significance analysis of microarray transcript levels in time series experiments. BMC Bioinform 8:1

Ernst J, Bar-Joseph Z (2006) STEM: a tool for the analysis of short time series gene expression data. BMC Bioinform 7:191. doi:10. 1186/1471-2105-7-191

Eynck C, Koopmann B, Karlovsky P, Von Tiedemann A (2009) Internal resistance in winter oilseed rape inhibits systemic spread of the vascular pathogen Verticillium longisporum. Phytopathology 99:802-811. doi:10.1094/PHYTO-99-7-0802

Fich EA, Segerson NA, Rose JKC (2016) The plant polyester cutin: biosynthesis, structure, and biological roles. Annu Rev Plant Biol 67:207-233. doi:10.1146/annurev-arplant-043015-111929

Flajšman M, Radišek S, Javornik B (2017) Pathogenicity assay of Verticillium nonalfalfae on hop plants. Bio-protocol 7:e2171. doi:10.21769/BioProtoc.2171

Fradin EF, Zhang Z, Juarez Ayala JC, Castroverde CDM, Nazar RN, Robb J, Liu C-M, Thomma BPHJ (2009) Genetic dissection of verticillium wilt resistance mediated by tomato Ve1. Plant Physiol 150:320-332. doi:10.1104/pp.109.136762

Gayoso C, Pomar F, Novo-Uzal E, Merino F, de Ilarduya OM (2010) The Ve-mediated resistance response of the tomato to Verticillium dahliae involves $\mathrm{H}_{2} \mathrm{O}_{2}$, peroxidase and lignins and drives PAL gene expression. BMC Plant Biol 10:232. doi:10.1186/ 1471-2229-10-232

Gharbi Y, Alkher H, Triki MA, Barkallah M, Emna B, Trabelsi R, Fendri I, Gdoura R, Daayf F (2015) Comparative expression of genes controlling cell wall-degrading enzymes in Verticillium dahliae isolates from olive, potato and sunflower. Physiol Mol Plant Pathol 91:56-65. doi:10.1016/j.pmpp.2015.05.006

Guo S, Zuo Y, Zhang Y, Wu C, Su W, Jin W, Yu H, An Y, Li Q (2017) Large-scale transcriptome comparison of sunflower genes responsive to Verticillium dahliae. BMC Genomics 18:42. doi:10.1186/s12864-016-3386-7

Haunold A (2010) Hops and hop growing. In: Verheye WH (ed) Soils, plant growth and crop production, vol 2. EOLSS Publishers Co Ltd, Oxford, pp 192-207

Hill ST, Sudarsanam R, Henning J, Hendrix D (2017) HopBase: a unified resource for Humulus genomics. Database (Oxford) 2017(1). doi:10.1093/database/bax009

Hu J, Barlet X, Deslandes L, Hirsch J, Feng DX, Somssich I, Marco Y (2008) Transcriptional responses of Arabidopsis thaliana during wilt disease caused by the soil-borne phytopathogenic bacterium Ralstonia solanacearum. PLoS ONE 3:e2589. doi:10.1371/ journal.pone.0002589

Hutson RA, Smith IM (1983) The response of tomato seedling roots to infection by Verticillium albo-atrum or Fusarium oxysporum f. sp. lycopersici. Annal Appl Biol 102:89-97. doi:10.1111/j. 1744-7348.1983.tb02668.x

Jakše J, Čerenak A, Radišek S, Satovic Z, Luthar Z, Javornik B (2013) Identification of quantitative trait loci for resistance to Verticillium wilt and yield parameters in hop (Humulus lupulus L.). Theor Appl Genetics 126:1431-1443. doi:10.1007/s00122013-2062-4 
Kirkwood JS, Legette LL, Miranda CL, Jiang Y, Stevens JF (2013) A metabolomics-driven elucidation of the anti-obesity mechanisms of xanthohumol. J Biol Chem 288:19000-19013. doi:10.1074/ jbc.M112.445452

Klosterman SJ, Anchieta A, Garcia-Pedrajas MD, Maruthachalam K, Hayes RJ, Subbarao KV (2011) SSH reveals a linkage between a senescence-associated protease and Verticillium wilt symptom development in lettuce (Lactuca sativa). Physiol Mol Plant Pathol 76:48-58. doi:10.1016/j.pmpp.2011.05.003

Li J, Zeng L, Xie J, Yue Z, Deng H, Ma X, Zheng C, Wu X, Luo J, Liu M (2015) Inhibition of osteoclastogenesis and bone resorption in vitro and in vivo by a prenylflavonoid xanthohumol from hops. Sci Rep 5:17605. doi:10.1038/srep17605

Livak KJ, Schmittgen TD (2001) Analysis of relative gene expression data using real-time quantitative PCR and the 2- $\Delta \Delta \mathrm{Ct}$ Method. Methods 25:402-408. doi:10.1006/meth.2001.1262

Mandelc S, Timperman I, Radišek S, Devreese B, Samyn B, Javornik B (2013) Comparative proteomic profiling in compatible and incompatible interactions between hop roots and Verticillium albo-atrum. Plant Physiol Biochem 68:23-31. doi:10.1016/j. plaphy.2013.03.017

Markakis EA, Tjamos SE, Antoniou PP, Roussos PA, Paplomatas EJ, Tjamos EC (2010) Phenolic responses of resistant and susceptible olive cultivars induced by defoliating and nondefoliating Verticillium dahliae pathotypes. Plant Dis 94:1156-1162. doi:10. 1094/PDIS-94-9-1156

Natsume S, Takagi H, Shiraishi A, Murata J, Toyonaga H, Patzak J, Takagi M, Yaegashi H, Uemura A, Mitsuoka C, Yoshida K, Krofta K, Satake H, Terauchi R, Ono E (2015) The draft genome of hop (Humulus lupulus), an essence for brewing. Plant Cell Physiol 56(3):428-441

Radišek S, Jakše J, Simončič A, Javornik B (2003) Characterization of Verticillium albo-atrum field isolates using pathogenicity data and AFLP analysis. Plant Dis 87(6):633-638. doi:10.1094/pdis. 2003.87.6.633

Radišek S, Jakše J, Javornik B (2006) Genetic variability and virulence among Verticillium albo-atrum isolates from hop. Eur J Plant Pathol 116:301-314

Ralhan A, Schottle S, Thurow C, Iven T, Feussner I, Polle A, Gatz C (2012) The vascular pathogen Verticillium longisporum requires a jasmonic acid-independent COI1 function in roots to elicit disease symptoms in Arabidopsis shoots. Plant Physiol 159:1192-1203

Reusche M, Thole K, Janz D, Truskina J, Rindfleisch S, Drübert C, Polle A, Lipka V, Teichmann T (2012) Verticillium infection triggers VASCULAR-RELATED NAC DOMAIN7-dependent de novo xylem formation and enhances drought tolerance in Arabidopsis. Plant Cell 24:3823-3837

Robb J, Shittu H, Soman KV, Kurosky A, Nazar RN (2012) Arsenal of elevated defense proteins fails to protect tomato against Verticillium dahliae. Planta 236:623-633. doi:10.1007/s00425012-1637-7

Robinson MD, Oshlack A (2010) A scaling normalization method for differential expression analysis of RNA-seq data. Genome Biol 11:R25. doi:10.1186/gb-2010-11-3-r25

Robinson MD, McCarthy DJ, Smyth GK (2010) edgeR: a bioconductor package for differential expression analysis of digital gene expression data. Bioinformatics 26:139-140. doi:10.1093/ bioinformatics/btp616

Roos J, Bejai S, Mozuraitis R, Dixelius C (2015) Susceptibility to Verticillium longisporum is linked to monoterpene production by TPS23/27 in Arabidopsis. Plant J 81:572-585. doi:10.1111/tpj. 12752
Sanavia T, Finotello F, Di Camillo B (2015) FunPat: function-based pattern analysis on RNA-seq time series data. BMC Genomics 16:S2. doi:10.1186/1471-2164-16-S6-S2

Song Y, Zhang Z, Seidl MF, Majer A, Jakse J, Javornik B, Thomma BP (2016) Broad taxonomic characterization of Verticillium wilt resistance genes reveals an ancient origin of the tomato Ve1 immune receptor. Mol Plant Pathol 18:195-209. doi:10.1111/ mpp. 12390

Štajner N, Cregeen S, Javornik B (2013) Evaluation of reference genes for RT-qPCR expression studies in hop (Humulus lupulus L.) during infection with vascular pathogen Verticillium alboatrum. PLoS ONE 8:e68228. doi:10.1371/journal.pone.0068228

Steenackers B, De Cooman L, De Vos D (2015) Chemical transformations of characteristic hop secondary metabolites in relation to beer properties and the brewing process: a review. Food Chem 172:742-756. doi:10.1016/j.foodchem.2014.09.139

Sun Q, Jiang H, Zhu X, Wang W, He X, Shi Y, Yuan Y, Du X, Cai Y (2013) Analysis of sea-island cotton and upland cotton in response to Verticillium dahliae infection by RNA sequencing. BMC Genomics 14:852. doi:10.1186/1471-2164-14-852

Tan G, Liu K, Kang J, Xu K, Zhang Y, Hu L, Zhang J, Li C (2015) Transcriptome analysis of the compatible interaction of tomato with Verticillium dahliae using RNA-sequencing. Front Plant Sci 6:428. doi: $10.3389 /$ fpls. 2015.00428

Thatcher LF, Manners JM, Kazan K (2009) Fusarium oxysporum hijacks COI1 \mbox-mediated jasmonate signaling to promote disease development in Arabidopsis. Plant J 58:927-939. doi:10. 1111/j.1365-313x.2009.03831.x

Toueni M, Ben C, Le Ru A, Gentzbittel L, Rickauer M (2016) Quantitative resistance to Verticillium wilt in Medicago truncatula involves eradication of the fungus from roots and is associated with transcriptional responses related to innate immunity. Front Plant Sci 7:1431. doi:10.3389/fpls.2016.01431

Untergasser A, Cutcutache I, Koressaar T, Ye J, Faircloth BC, Remm M, Rozen SG (2012) Primer3 - new capabilities and interfaces. Nucleic Acids Res 40:e115

Van Cleemput M, Cattoor K, De Bosscher K, Haegeman G, De Keukeleire D, Heyerick A (2009) Hop (Humulus lupulus)derived bitter acids as multipotent bioactive compounds. J Nat Prod 72:1220-1230. doi:10.1021/np800740m

van Esse HP, Fradin EF, de Groot PJ, de Wit PJGM, Thomma BPHJ (2009) Tomato transcriptional responses to a foliar and a vascular fungal pathogen are distinct. Mol Plant Microbe Interact 22:245-258. doi:10.1094/MPMI-22-3-0245

Wasternack C, Song S (2016) Jasmonates: biosynthesis, metabolism, and signaling by proteins activating and repressing transciption. J Exp Bot erw443. doi:10.1093/jxb/erw443

Xu L, Zhu L, Tu L, Liu L, Yuan D, Jin L, Long L, Zhang X (2011) Lignin metabolism has a central role in the resistance of cotton to the wilt fungus Verticillium dahliae as revealed by RNA-Seqdependent transcriptional analysis and histochemistry. J Exp Bot 62:5607-5621. doi:10.1093/jxb/err245

Yadeta KA, Thomma BP (2013) The xylem as battleground for plant hosts and vascular wilt pathogens. Front Plant Sci 4:97. doi:10. 3389/fpls.2013.00097

Zhou X, Bao S, Liu J, Zhuang Y (2016) De novo sequencing and analysis of the transcriptome of the wild eggplant species Solanum aculeatissimum in response to Verticillium dahliae. Plant Mol Biol Rep 34:1193-1203. doi:10.1007/s11105-0160998-7 